Journal of Analytical Chromatography and Spectroscopy (2018) Volume 1 doi:10.24294/jacs.v1i2.774

\title{
Investigation of Thermometrical Optical Characteristics Influence on Spectral Thermometry Metrological Characteristics
}

\author{
Leonid Zhukov' ${ }^{1}$ D. A. Petrenko² , A. L. Kornienko ${ }^{3}$
}

${ }^{1}$ Department of Thermometry and Physics-Chemical Investigations, Physics-Technological Institute of Metals and Alloys of Ukraine National Academy of Sciences, Kiev, Ukraine

${ }^{2}$ Junior research fellow, Physics-Technological Institute of Metals and Alloys of Ukraine National Academy of Sciences

${ }^{3}$ Research fellow, Physics-Technological Institute of Metals and Alloys of Ukraine National Academy of Sciences

\begin{abstract}
The complex of investigations of optical characteristics of controlled objects and spectral symmetric-wave and two-colour compensating thermometry (SWT and TCCT) influence on their errors of method and instrumental errors is performed. Advantages of these new methods in the field of errors of method, in comparison with known spectral and also classical energy and spectral ratio thermometry are proved. It is established that right use of the SWT and the TCCT, which takes into account thermometrical conditions, allows to completely exclude methodical and instrumental components from the errors of optical temperature measurements. Spectral thermometry is based on remote quantitative determination of the spectral emissivity distributions of controlled objects. Using these distributions and well-known relationships between the spectral coefficients of emission, absorption, reflection and transmission we can determine macro-optical characteristics of these objects and remotely investigate them.
\end{abstract}

Keywords: Temperature; Spectral thermometry; Spectral Distribution of Emissivity; Errors of Method; Instrumental Errors

\section{Introduction}

Classical optical thermometry technologies can be used on the opened ensuring optical contact controlled objects without temperature corrections only in "black body" case for the one-colour and "black and grey body" case for two-colour pyrometers. Under other conditions the optical thermometry possibilities are limited with well-known problems of the unknown and non-stable emissivity and transmissivity. At the same time, regardless of these showed shortcomings, optical thermometry has no alternative for the most effective continuous temperature control, including the temperature measurements of small, moving, remote and high-temperature objects. Therefore in the last years, versus classical thermometry, investigations have been directed on more perfect spectral thermometry technologies. Spectral thermometry allows to solve main task - to decrease or even to exclude errors of method in optical thermometry. However, excluding errors of method spectral thermometry receive other problem - instrumental errors problem. This article is devoted to new spectral thermometry technologies which allow to exclude errors of method and simultaneously receive good results for instrumental errors.

\section{State and place of classical optical and spectral thermometry in temperature measurements}

Production processes, first of all used in metallurgy, are developed on temperature action on raw an auxiliary materials. Temperature is the main parameter here which determines ready product quality and expenses for its manufac-

\footnotetext{
Copyright (C) 2018 Leonid Zhukov et al.

doi: 10.24294/jacs.v1i2.774

EnPress Publisher LLC.This work is licensed under the Creative Commons Attribution-NonCommercial 4.0 International License (CC BY-NC 4.0).

http://creativecommons.org/licenses/ by/4.0/
} 
ture. That is why in metrological support structure of the most representative in metrological culture Japanese enterprises Kawasaki Seitetsu thermometry dominates, constitutes about $30 \%{ }^{[1]}$ and exceeds manyfold the number of other widely-used measurements, for example, in 6 times - chemical composition and in 8 times - mass (Figure 1).

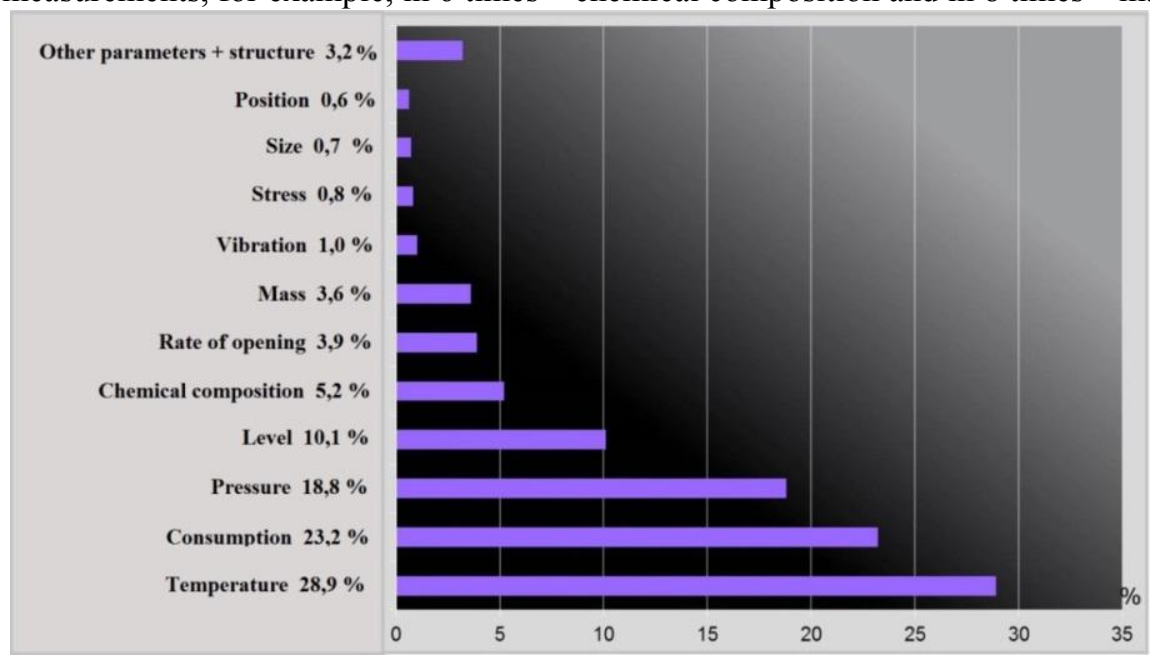

Figure 1; Metrological support structure of metallurgical enterprises Kawasaki Seitetsu (Japan).

In industry thermoelectrical and optical technologies are used for medium and high temperature measurements. Now thermoelectrical thermometer is the best instrument, but only for periodical measurements.

However, the most effective, providing optimal control of production processes in the "on-line" regime is continuous temperature control. Thus, developed by PTIMA NASU on the base of continuous light-guide temperature measurement Production Control System for induction melting, processing and pouring of metal reduces power consumption in 2 times and waste of charge materials- on 30\%, increases lining life duration in 2,5 times and furnaces productivity - on $40 \%$, excludes spoilage "due to the temperature" and lining breaks connected with uncontrolled overheating or overcooling of metal in furnaces. On the other hand the world expenses for the development of continuous temperature control of the convertor process in metallurgy have already exceeded 1 billion USD. According to the estimations of Research \& Development Center of Nippon Steel Corporation, these expenses now amount not less than 1,5 billion USD. Nevertheless, this task has not yet been solved and convertor process is controlled periodically with thermoelectrical changeable transducers of short-time immersion. The above quantitative indices, on the one hand, emphasize a high technical and economic efficiency and on the other hand - exceptional complexity and topicality of continuous temperature control. For such control optical thermometry has no alternative. Therefore, it is no accident that the first optical thermometers were developed and appeared precisely in metallurgy.

Optical thermometry is based on Kirchhoff, Lambert, Relay-Jeans, Wien, Plank, Stephan-Boltzmann and Lambert thermodynamic laws of heat radiation. In the following after discovering years, these laws have been further developed. Currently, there are more than 60 of their formulations, describing the various parameters of the interaction of thermal electromagnetic radiation with matter under conditions of thermodynamic equilibrium.

Until the early 80-th of last century the "classical" energy and spectral ratio pyrometry technologies were intensively investigated. As a result of these researches metrological characteristics of the optical thermometry were significantly improved and the field of it use was greatly widened. Nowadays, as for instrumental errors and other characteristics, the optical thermometry is not inferior, and even exceeds thermoelectrical thermometry and therefore is widely used for reference temperature measurement and construction of the thermodynamic and practical temperature scales. Classical optical thermometry technologies can be used on available for pyrometers controlled objects with stable radiation characteristics and transmissivity of the intermediate medium. Without use temperature corrections it is possible in the case of a "black" body for energy pyrometry and the cases of "black" and "grey" bodies for spectral ratio pyrometry or with use corrections - for any coloured bodies. It can be concluded that with increasing of the number of operating waves optical thermometry capabilities to reduce errors of method significantly widen. In industry, when 
emissivity and transmissivity of intermediate medium accidentally change, classical thermometry technologies use is sharply limited by a high errors of method. Under such conditions errors of method of classical thermometry exceed hundred degrees in absolute form. For example, for tungsten with temperature $T=1600 \mathrm{~K}$ for $\lambda_{1}=0,7 \mu \mathrm{m}$ errors of energy one-colour thermometry achieve $97,1 \mathrm{~K}(6,07 \%)$ and for $\lambda_{2}=0,9 \mu \mathrm{m}-137,7 \mathrm{~K}(8,42 \%)$ and of two-colour for $\lambda_{\text {eq }} \lambda_{1} \cdot \lambda_{2} / \lambda_{2}-\left(\lambda_{2}-\lambda_{1}\right)=3,15 \mu \mathrm{m}-48,2 \mathrm{~K}(3,01 \%)^{[2]}$. That is why, in the last years, investigations have been directed on more perfect spectral (multicolour) thermometry technologies. They provide a significant increase of metrological characteristics of the optical thermometry under conditions of unknown and accidentally changing characteristics of heat radiation and transmission of electromagnetic energy. The most significant results were obtained in Belarus, Germany, Japan, Russia, Ukraine and the USA.

The rapid development and miniaturization of optical and electronic technologies, including optoelectronic, fiber-optical, microprocessor and computer, also stimulates the development of spectral thermometry.

There are two physical material substances, i.e., matter (controlled object) and its electromagnetic field (thermal radiation) to be operated in optical thermometry. Heat is transferred by radiation, therefore radiation has the energy, entropy and temperature. The conceptions of the temperature and spectral distribution function of heated body radiation were a significant success of the new physics of the first half of the last century. In the case of the absolute black body these substances are in thermodynamic equilibrium and so their temperatures are equal. This property of the absolute black body and its radiation is used in optical thermometry for reference measurements, construction of temperature scales and calibration of optical thermometers.

Optical thermometry was originating simultaneously with development of thermal radiation theory, when the concept and the term temperature of radiation was not used, and therefore concepts and terms "conditional temperatures" or "pseudotemperatures" of controlled objects were introduced. They do not correspond to the physical sense and definitions of the temperature in thermodynamics, molecular-kinetic theory and statistical physics. For example, if we consider fundamental for temperature determination direction of heat transfer by radiation, the body with less "pseudotemperature" under certain conditions, can transfer energy and heat the body with higher "pseudotemperature". This contradicts the definition of temperature in physics, which sets the direction of heat transfer. Therefore, Prof. L. F. Zhukov at the time suggested to use more natural in the optical thermometry and physically based term of the "temperature of radiation". The authors also use term "temperature of radiation" in this article.

Started by scientific thermometric society discussion about possibilities of the optical thermometry, has not yet been finished. Are the transferred by radiation information about controlled objects, enough for their temperature measurements? The positive and negative answers on this fundamental question of modern optical thermometry divided experts into two opposite camps. Recent achievements of spectral thermometry develop events in it good and don't leave any hope for skeptics.

The results of investigations carried out by the authors in the most perspective areas of spectral thermometry symmetric-wave (SWT) and two-colour compensating (TCCT) thermometry are presented in this article. The Department of Thermometry of the PTIMA NASU is also developing other spectral thermometry technologies, including spectral-compensating and parabolic approximation. These technologies significantly improve the metrological characteristics of developed by us continuous contactless and light-guide temperature control in metallurgy, ceramic, glass, energy and other industries.

The main developers, the number of their patents and some characteristics of developed spectral thermometry technologies are presented at the Table 1.

\begin{tabular}{|l|l|l|l|l|}
\hline Country & Developer & $\begin{array}{l}\text { Number of } \\
\text { patents }\end{array}$ & $\begin{array}{l}\text { Number of } \\
\text { operating waves }\end{array}$ & Relative error, \% \\
\hline Ukraine & PTIMA NAS of Ukraine & 18 & 3 & $<0,5$ \\
\hline
\end{tabular}




\begin{tabular}{|l|l|l|l|l|}
\hline USA & $\begin{array}{l}\text { Princeton University \& } \\
\text { NASA }\end{array}$ & 11 & $3-8$ \\
\hline Belarus & $\begin{array}{l}\text { Institute of physics named after B. I. } \\
\text { Stepanov NAS of Belarus }\end{array}$ & 4 & 3 and more & 2 \\
\hline Russia & $\begin{array}{l}\text { Corporate enterprise } \\
\text { «Scientific-research institute of } \\
\text { optical-electronic instrument } \\
\text { engineering» }\end{array}$ & 6 & 8 & N/A \\
\hline Japan & $\begin{array}{l}\text { Noritake Co., Ltd. Erich Weinert" betrieb des } \\
\text { kombinates }\end{array}$ & 3 & 3 & N/A \\
\hline Germany
\end{tabular}

Table 1. The main developers of the spectral thermometry technologies

They have developed different types and construction of auxiliary devices, including tuyeres, light-guide and cooling units, units of optical joint, etc. These devices successfully solve tasks for their purpose. Therefore, exclusion of influence of emissivity unknown values and their accidental changes on optical thermometry errors of method remains the main problem.

\section{Classification, qualitative and quantitative description of spectral emissivity distributions}

To provide presented below investigations spectral distributions of metal alloys emissivity are studied and classified. Depending on the surface state, radiative characteristics of metal alloys qualitatively and quantitatively change in wide ranges. The state of radiative surface is determined with physics-chemical processes in alloy and interaction between it, atmosphere and lining. Intensity and direction of these processes are depended on the temperature and chemical composition of alloy and lining.

Spectral distributions of metals emissivity, for example, tungsten, are studied in ideal conditions (in vacuum or inert gases) and presented in metal optics and thermometry well and in detail ${ }^{[3]}$. Most of metallurgical processes, including obtaining, processing and pouring of liquid metal are performed in usual atmosphere. During these processes radiative surface of controlled metal can be clean, covered by semitransparent in visible and near infrared ranges of spectrum oxide films or oxidized, as well as covered by slag. Metal has such surface, for example, during tapping from metallurgical furnaces and aggregates and also under mold during continuous pouring. That is why obtained in metal optics results cannot be used in thermometry under these industrial conditions. For example, these distributions for clean and covered with oxide films surface can be respectively "metal", "grey" or increasing "non-metal" state during increase of films thickness ${ }^{[4,5]}$. Non-metal inclusions influence essentially on spectral distributions of emissivity too.

Classification of spectral distributions of emissivity of metal alloys under industrial conditions is presented at the Table 2 and Figure $2^{[6]}$.

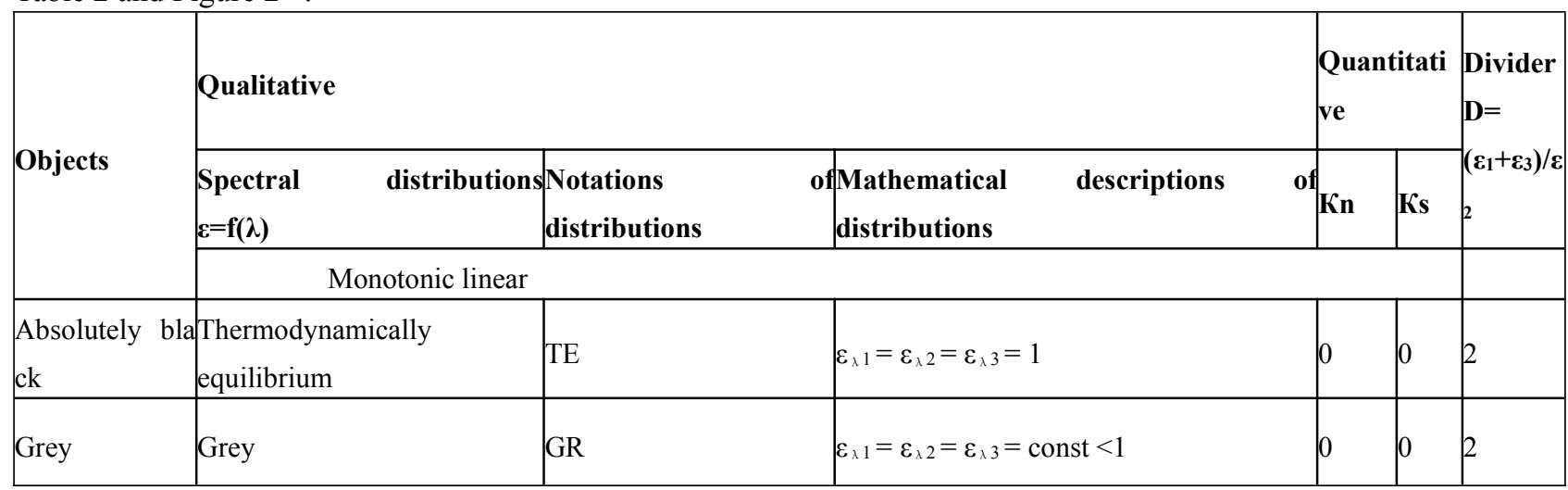




\begin{tabular}{|c|c|c|c|c|c|c|}
\hline \multirow{7}{*}{ Coloured } & Decreasing linear & DL & $1 \geq \varepsilon_{\lambda 1}>\varepsilon_{\lambda 2}>\varepsilon_{\lambda 3}$ & 0 & $<0$ & 2 \\
\hline & Increasing linear & IL & $\varepsilon_{\lambda 1}<\varepsilon_{\lambda 2}<\varepsilon_{\lambda 3} \leq 1$ & 0 & $>0$ & 2 \\
\hline & \multicolumn{5}{|c|}{ Monotonic nonlinear } & \\
\hline & Decreasing convex & DCX & $1 \geq \varepsilon_{\lambda 1}>\varepsilon_{\lambda 2}>\varepsilon \lambda_{3}$ & $>0$ & $<0$ & $\mathrm{D}<2$ \\
\hline & Decreasing incurved & DIC & $1 \geq \varepsilon_{\lambda 1}>\varepsilon_{\lambda 2}>\varepsilon \lambda_{3}$ & $<0$ & $<0$ & $\mathrm{D}>2$ \\
\hline & Increasing convex & ICX & $\boldsymbol{\varepsilon}_{\lambda 1}<\boldsymbol{\varepsilon}_{\lambda 2}<\boldsymbol{\varepsilon}_{\lambda 3} \leq 1$ & $>0$ & $>0$ & $\mathrm{D}<2$ \\
\hline & Increasing incurved & IIC & $\boldsymbol{\varepsilon}_{\lambda 1}<\boldsymbol{\varepsilon}_{\lambda 2}<\boldsymbol{\varepsilon}_{\lambda 3} \leq 1$ & $<0$ & $>0$ & $\mathrm{D}>2$ \\
\hline
\end{tabular}

Table 2. Spectral optical characteristics of metal alloys under temperature control

Depending on the state of the radiative surface of most widespread in metallurgy iron-carbon alloys and conditions of temperature control, these distributions can be thermodynamically equilibrium (TE), grey (GR) or coloured decreasing (DL, DCX, DIC), increasing (IL, ICX, IIC), linear (DL, IL), convex (DCX, ICX) and incurved (DIC, IIC). These distributions change quantitatively and qualitatively in wide ranges and significantly overlap spectral radiative characteristics of other materials, for example, ceramic, glass, etc.

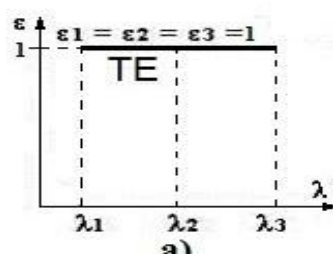

a)

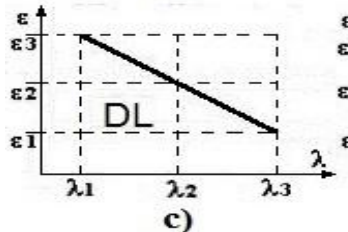

c)

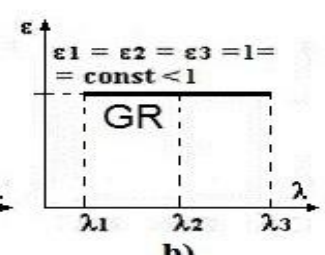

b)

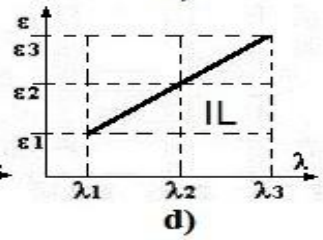

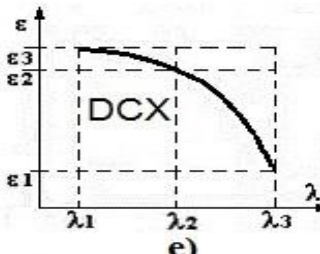

e)

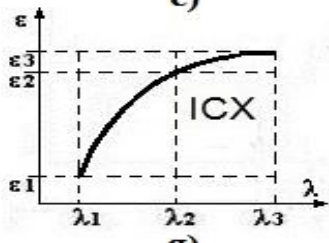

g)
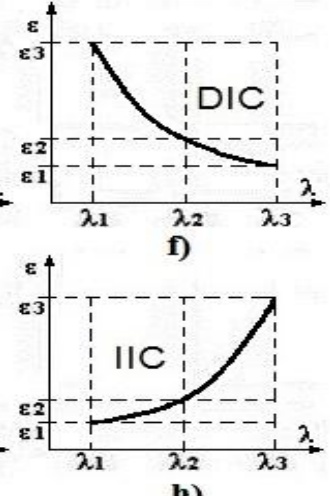

Figure 2. Spectral distributions of emissivity

Quantitative characteristics of classified spectral distributions of emissivity also are presented at the Table 2 . In principle, distributions can be quantitatively determined using the coefficient of nonlinearity $K n$, the coefficient of steepness $K s$ and quantitative values of emissivity, which are not shown at the Table 2 and Figure 2. The dimensionless $K n$ is calculated according to the next formula

$K n=\varepsilon_{2}-\varepsilon_{2 l}$,

where $\varepsilon_{2 l}$-value of the emissivity on wave $\lambda_{2}$ for linearly approximated distribution $\varepsilon_{1}=f(\lambda)$.

$K s$ is determined according to the formula

$K s=\left(\varepsilon_{3}-\varepsilon_{1}\right) /\left(\lambda_{3}-\lambda_{1}\right), \mathrm{m}^{-1}$,

where $\varepsilon_{3}$ and $\varepsilon_{1}$ - values of the emissivity on waves $\lambda_{3}$ and $\lambda_{1}$, respectively.

\section{Main idea, physical bases and common pyrometric equation of the SWT}

Idea of spectral SWT is based on determined by the authors correlation between emissivity value in average part of operating range of spectrum and emissivity values on symmetrically placed boundary parts of the spectrum ${ }^{[7]}$. This statement is correct for controlled objects in condensed state, including metal alloys.

To determine the temperature $T$ of the controlled object, for example, clean surface of iron-carbon alloy with DCX-distribution (Figure 2, e), it is necessary to measure at least 3 one-colour radiation temperatures $S_{1}, S_{2}$ and $S_{3}$ on symmetrically placed 3 operating waves $\lambda_{1}, \lambda_{2}$ and $\lambda_{3}$. Radiation temperatures, according to the Wien law, are connected with the $T$ with system of 3 pyrometric equations via known operating waves $\lambda_{1}, \lambda_{2}$ and $\lambda_{3}$, the second Plank constant $\mathrm{C} 2=0,014388 \cdot \mathrm{m}$ and appropriate values of emissivity $\varepsilon_{1}, \varepsilon_{2}$ and $\varepsilon_{3}$. 


$$
\left\{\begin{array}{l}
\frac{1}{T}-\frac{1}{S_{1}}=\frac{\lambda_{1}}{C_{2}} \ln \left(\varepsilon_{1}\right) \\
\frac{1}{T}-\frac{1}{S_{2}}=\frac{\lambda_{2}}{C_{2}} \ln \left(\varepsilon_{2}\right) \\
\frac{1}{T}-\frac{1}{S_{3}}=\frac{\lambda_{3}}{C_{2}} \ln \left(\varepsilon_{3}\right)
\end{array}\right.
$$

The system cannot be solved because it has 4 unknown quantities: $T, \varepsilon_{1}, \varepsilon_{2}$ and $\varepsilon_{3}$. Symmetric placing of operating waves and established by us correlation provide apriori information about $\varepsilon_{2}, \varepsilon_{1}$ and $\varepsilon_{3}$. For example, for concerned DL-, IL-, GR- and TE-distributions of emissivity (see the Table 2) $\varepsilon_{2}=\left(\varepsilon_{1}+\varepsilon_{3}\right) / 2$. From equations (1) and (3) of the system we can obtain formulas for $\varepsilon_{1}$ and $\varepsilon_{3}: \varepsilon_{1}=\exp \left(-\frac{C_{2}\left(T-S_{1}\right)}{T \lambda_{1} S_{1}}\right)$ and $\varepsilon_{3}=\exp \left(-\frac{C_{2}\left(T-S_{3}\right)}{T \lambda_{3} S_{3}}\right)$

After substitution $\varepsilon_{1}$ and $\varepsilon_{3}$ in $\varepsilon_{2}=\left(\varepsilon_{1}+\varepsilon_{3}\right) / 2$ the next formula can be obtained

$\varepsilon_{2}=\frac{\varepsilon_{1}+\varepsilon_{3}}{2}=\frac{\exp \left(-\frac{C_{2}\left(T-S_{1}\right)}{T \lambda_{1} S_{1}}\right)+\exp \left(-\frac{C_{2}\left(T-S_{3}\right)}{T \lambda_{3} S_{3}}\right)}{2}$.

Then we use it in equation (2) of the system

$\frac{1}{T}-\frac{1}{S_{2}}=\frac{\lambda_{2}}{C_{2}} \ln \left(\varepsilon_{2}\right)=\frac{\lambda_{2}}{C_{2}} \ln \left(\frac{\exp \left(-\frac{C_{2}\left(T-S_{1}\right)}{T \lambda_{1} S_{1}}\right)+\exp \left(-\frac{C_{2}\left(T-S_{3}\right)}{T \lambda_{3} S_{3}}\right)}{2}\right)$

and derive common pyrometric equation (mathematical model) of the SWT for temperature calculation of the objects with linear spectral distributions of emissivity

$$
\frac{\lambda_{2}}{C_{2}} \ln \left(\frac{\exp \left(-\frac{C_{2}\left(T-S_{1}\right)}{T \lambda_{1} S_{1}}\right)+\exp \left(-\frac{C_{2}\left(T-S_{3}\right)}{T \lambda_{3} S_{3}}\right)}{2}\right)-\frac{1}{T}+\frac{1}{S_{2}}=0 .
$$

For nonlinear DCX- and ICX-distributions $\mathrm{D}<2$ and for DIC- and IIC-distributions $\mathrm{D}>2$.

Adequacy of the mathematical model (4) of linear symmetric-wave thermometry (LSWT) is confirmed with temperature calculations for linear radiators, including "grey" and thermodynamically equilibrium objects. Errors of method of the LSWT are equal to zero for such objects.

\section{Influence of optical characteristics of the controlled objects and SWT on its errors of method}

Complex of investigations of metal alloys and LSWT optical characteristics influence on its errors of method is performed. Spectral distribution of tungsten emissivity is used as DCX-distribution. This spectral distribution is the most studied in thermometry, metal optics and lighting engineering as well as tabulated in detail and quantitatively presented in literature ${ }^{[8]}$. It is used in optical thermometry, including spectral thermometry, for comparative investigations of new and known thermometry technologies. Also it the most fully corresponds to spectral distributions of iron-carbon alloys in quantitative and qualitative aspects. Appropriate mirror representations of DCX-distribution are used for DIC-, ICX- and IIC-distributions, i.e. DICmw-, ICXmw- and IICmw-distributions. The systematical error of presented in ${ }^{[8]}$ investigation results of tungsten emissivity in the spectral range $0,4-1,0 \mu \mathrm{m}$ equals $0,1-0,3 \%$, and the standard deviation $-0,5 \%$. The largest value of systematical error is obtained for wavelengths $0,4 \mu \mathrm{m}$ and $1,0 \mu \mathrm{m}$. It was established that the large differences between the experimental spectral distributions of the tungsten emissivity are not physically based and do not correspond to the changes of the electromagnetic characteristics of this metal depending on its temperature and radiation spectrum. The indicated differences can be explained first of all by the errors of the high-temperature experiments for the determination of the optical properties of tungsten. These experiments are difficult to realize. Therefore, we have approximated the available experimental data in the spectral range $0,3-1,5 \mu \mathrm{m}$ by the next 
more "smooth" the 6-power polynoms:

$$
\begin{gathered}
\varepsilon_{\text {DCXaw }}=0,0905875 \lambda_{\mathrm{n}}^{6}+0,6024 \lambda_{\mathrm{n}}^{5}-1,6045 \lambda_{\mathrm{n}}^{4}+2,2555 \lambda_{\mathrm{n}}^{3}-1,8327 \lambda_{\mathrm{n}}^{2}+0,6475 \lambda_{\mathrm{n}}-0,4234 ; \\
\varepsilon_{\text {ICXamw }}=-0,0906 \lambda_{\mathrm{n}}{ }^{6}+0,6024 \lambda_{\mathrm{n}}^{5}-1,6045 \lambda_{\mathrm{n}}^{4}+2,2555 \lambda_{\mathrm{n}}^{3}-1,8327 \lambda_{\mathrm{n}}^{2}-0,6475 \lambda_{\mathrm{n}}-0,4015 ; \\
\varepsilon_{\text {IICamw }}=0,0909875 \lambda_{\mathrm{n}}{ }^{6}-0,2693 \lambda_{\mathrm{n}}^{5}+0,2682 \lambda_{\mathrm{n}}^{4}-0,0177 \lambda_{\mathrm{n}}{ }^{3}-0,1144 \lambda_{\mathrm{n}}{ }^{2}-0,1443 \lambda_{\mathrm{n}}+0,5593 ; \\
\varepsilon_{\text {DICamw }}=-0,091 \lambda_{\mathrm{n}}^{6}+0,2693 \lambda_{\mathrm{n}}^{5}-0,2682 \lambda_{\mathrm{n}}^{4}+0,0177 \lambda_{\mathrm{n}}^{3}+0,1144 \lambda_{\mathrm{n}}{ }^{2}+0,1443 \lambda_{\mathrm{n}}+0,2656 ;
\end{gathered}
$$

This approximation allows to exclude a significant part of the mentioned above standard deviation. This excluded part reaches $0,3 \%$ in spectral range $0,3-1,5 \mu \mathrm{m}$.

The LSWT errors of method for non-linear spectral distributions of emissivity depend on the optical characteristics of the spectral thermometry and controlled objects, including average wavelength $\lambda_{2}=\left(\lambda_{1}+\lambda_{3}\right) / 2$, width of spectral range $\Delta \lambda=\left(\lambda_{3}-\lambda_{1}\right) / 2$ and also qualitative and quantitative characteristics of spectral emissivity distributions [6]. Figure 3 and 4 present dependences of the LSWT errors of method ( $\delta_{m . L S W T}$ ) on $\lambda_{2}$ and $\Delta \lambda$, respectively. The dependences are obtained for controlled objects with different spectral distributions of emissivity.
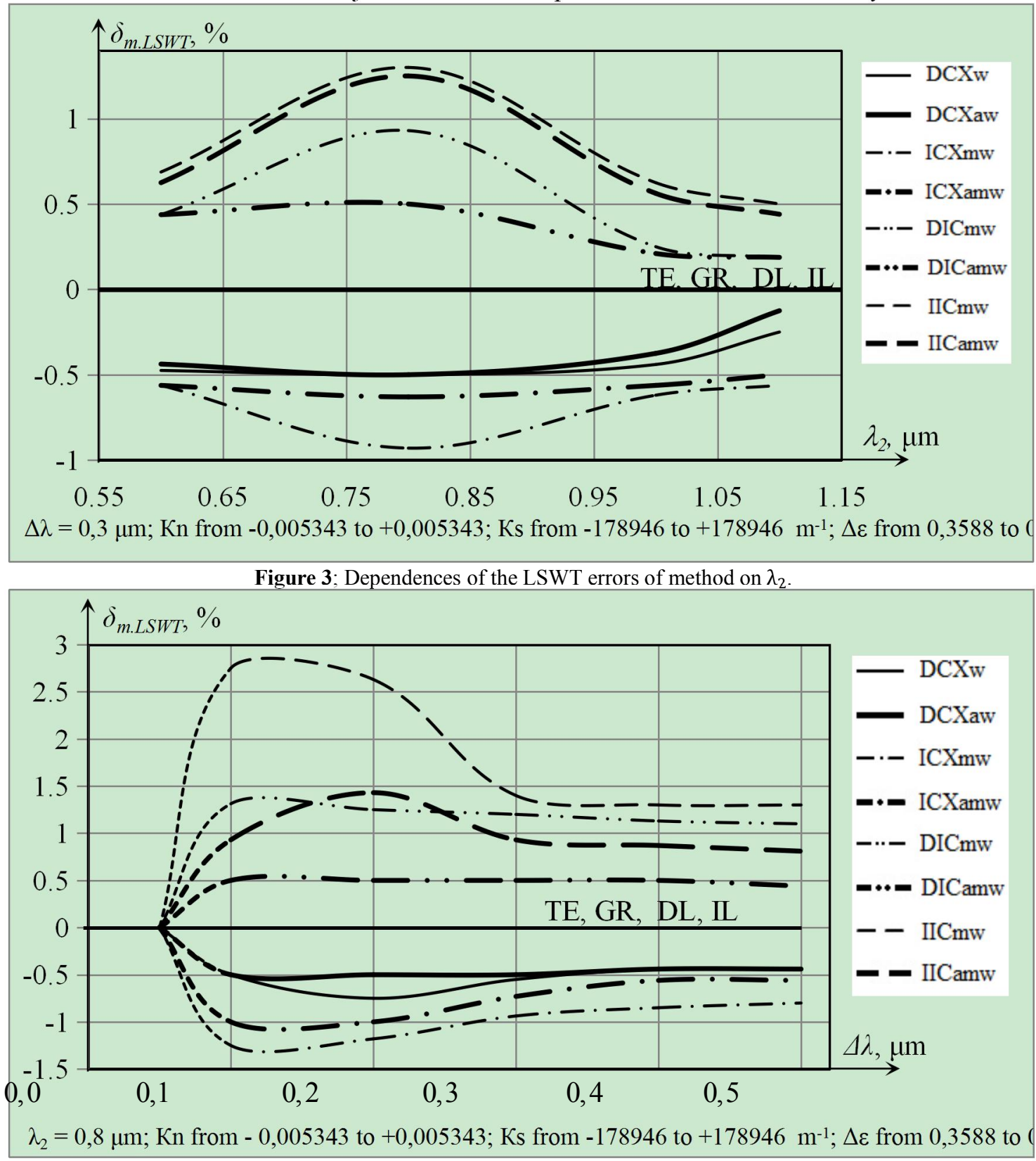
Figure 4; Dependences of the LSWT errors of method on $\Delta \lambda$.

The established regularities show that errors of method of the LSWT can be reduced respectively in 1,3-5,0 and 1,1-2,3 times by means of optimization of $\lambda_{2}$ and $\Delta \lambda$ values. For $\Delta \lambda \leq 0,05 \mu \mathrm{m}$ the errors can be completely excluded. This influence is determined by relationship between nonlinearity of emissivity spectral distributions and values of $\lambda_{2}$ and $\Delta \lambda$.

The influence of spectral optical characteristics of controlled objects on the LSWT errors of method is investigated. On the Figures 5 and 6 established influence regularities of emissivity (parallel, from initial spectral DCX-distribution of tungsten emissivity, $\Delta \varepsilon$-displacements on $\lambda_{1}$ and $\lambda_{3}$ ) and nonlinearity coefficient $K_{n}$ are presented ${ }^{[6]}$.

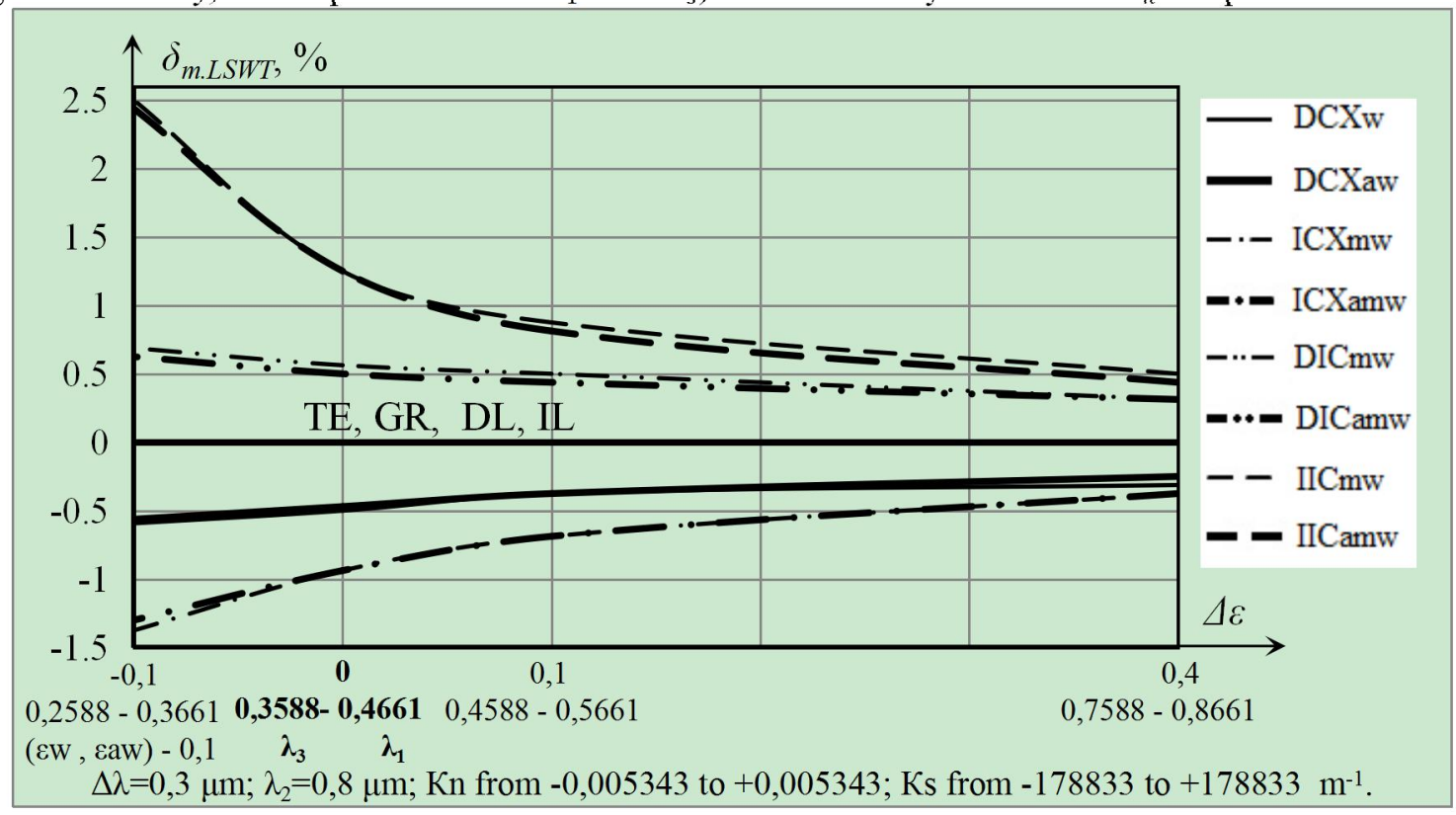

Figure 5; Dependences of the LSWT errors of method on $\Delta \varepsilon$.

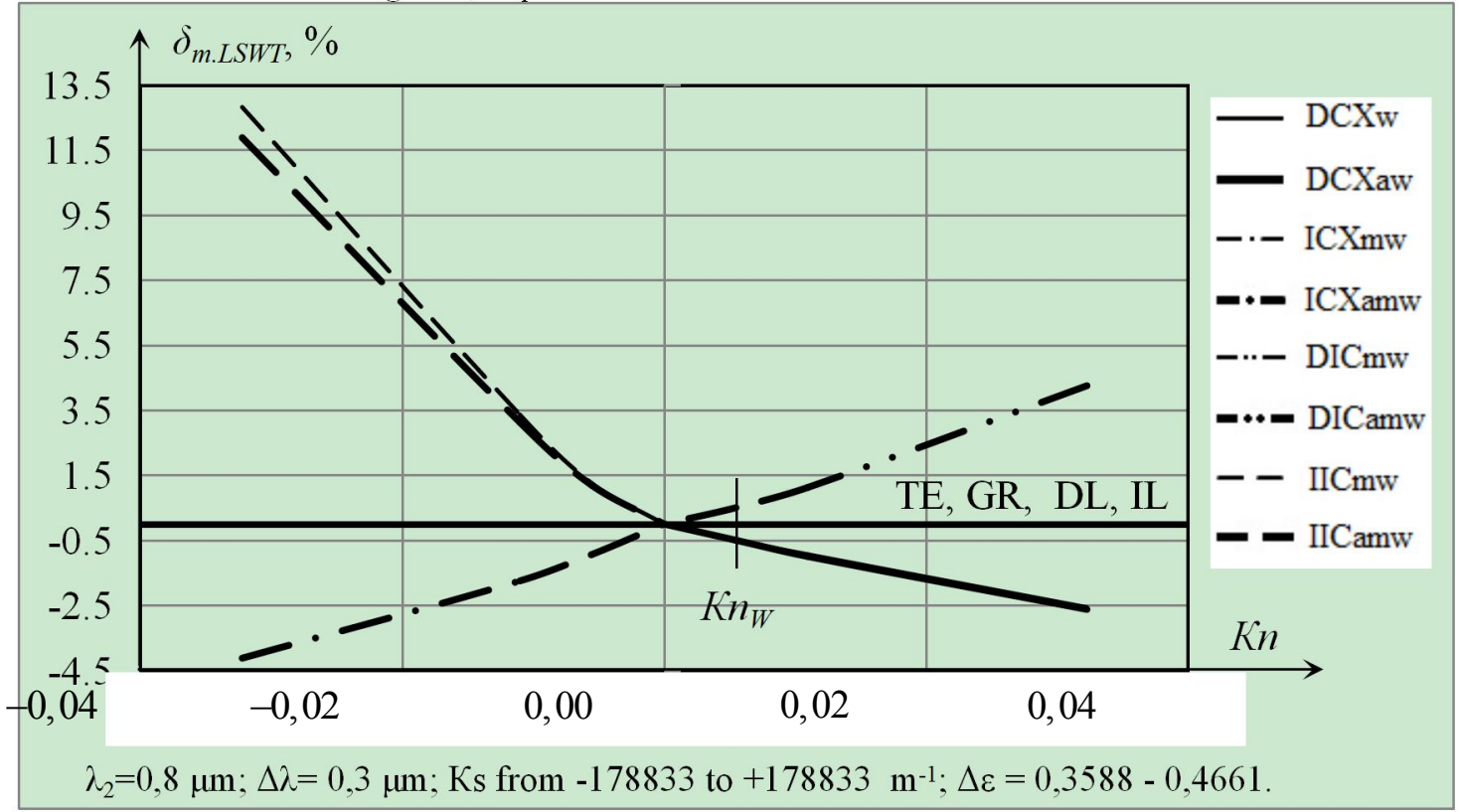

Figure 6; Dependences of the LSWT errors of method on Kn.

Strong influence of emissivity spectral distributions types, which is determined by emissivity values combinations with values of operating wavelengths, is proved. It is shown that errors are minimal for DCX-distributions of emissivity which are the most typical for iron-carbon alloys. Maximal errors are observed for IIC-distributions. Adequate in optical thermometry decrease of errors with emissivity increase is confirmed. Strong influence of $K_{n}$ on errors of the LSWT is shown. Such influence is not observed for $K_{s}$. It can be concluded from the Figure 5, that due to correct choosing of 
the controlled object, errors of method can be reduced in 1,4-7,8 times.

Established regularities (Figures 3-6) allow to reduce errors of method of the LSWT in some times or even completely exclude them for an appropriate values of $\Delta \lambda$. Universal method of the SWT excludes errors of method for any spectral distributions of emissivity ${ }^{[2]}$.

Thermometry technologies are based on remote quantitative determination of the spectral emissivity distributions of controlled objects. Using these distributions and well-known relationships between the spectral coefficients of radiation and absorption, reflection and transmission macro-optical characteristics of these objects can be determined and remote identification of them can be performed. Therefore, developed spectral thermometry technologies can also be used for special and aerospace researches and control.

\section{Main idea, physical bases and common pyrometric equation of the TCCT}

The TCCT is based on use of measured $S_{2 c}$ and mirror $S_{2 c}^{\prime}$ two-colour radiation temperatures of controlled object. Desired temperature of the object is calculated via these two-colour radiation temperatures ${ }^{[9]}$. Measured two-colour radiation temperature $S_{2 c}$ of alloy corresponds to real, for example, decreasing, spectral distribution of emissivity (RD). The mirror two-colour radiation temperature $S_{2 c}^{\prime}$ corresponds to mirror reflected distribution, i.e., increasing (MD). At the same time values of emissivity on operating waves $\lambda_{1}$ and $\lambda_{2}$ are on the levels $\varepsilon_{1}$ and $\varepsilon_{2}$, i.e. $\varepsilon_{R D}\left(\lambda_{1}\right)=\varepsilon_{M D}\left(\lambda_{2}\right)$ and $\varepsilon_{M D}\left(\lambda_{1}\right)=\varepsilon_{R D}\left(\lambda_{2}\right)$. For the TCCT realization inverse situation can also take place, i.e. real spectral distribution is increasing and mirror spectral distribution will be decreasing. In the cases of "black" and "grey" bodies real and mirror distributions are the same and $S_{2 c}=S_{2 c}^{\prime}=T$.

Measured two-colour radiation temperature $S_{2 c}$ is connected with the temperature $\mathrm{T}$ of the controlled object via appropriate values of emissivity $\varepsilon_{1}$ and $\varepsilon_{2}$

$$
\frac{1}{T}-\frac{1}{S_{2 c}}=\frac{\lambda_{e q}}{C_{2}} \ln \left(\frac{\varepsilon_{1}}{\varepsilon_{2}}\right)
$$

where $\lambda_{e q}=\frac{\lambda_{1} \lambda_{2}}{\lambda_{2}-\lambda_{1}}$ - equivalent wavelength.

Mirror two-colour radiation temperature $S_{2 c}^{\prime}$ is connected with the mentioned parameters of controlled object in a similar

$$
\frac{1}{S_{2 c}^{\prime}}-\frac{1}{T}=\frac{\lambda_{e q}}{C_{2}} \ln \left(\frac{\varepsilon_{1}}{\varepsilon_{2}}\right)
$$

Two-colour radiation temperatures $S_{2 c}$ and $S_{2 c}^{\prime}$ can be expressed via appropriate one-colour radiation temperatures on $\lambda_{1}$ and $\lambda_{2}$

$$
\begin{aligned}
S_{2 c} & =\frac{1}{\lambda_{e q}\left(\frac{1}{\lambda_{1} \cdot S_{1}}-\frac{1}{\lambda_{2} \cdot S_{2}}\right)} \\
S_{2 c}^{\prime} & =\frac{1}{\lambda_{e q}\left(\frac{1}{\lambda_{1} \cdot S_{1}^{\prime}}-\frac{1}{\lambda_{2} \cdot S_{2}^{\prime}}\right)} .
\end{aligned}
$$

It can be concluded from formulas $(5,6)$, that reciprocal values of $S_{2 c}$ and $S_{2 c}^{\prime}$ are displaced relatively reciprocal value of the $T$ on the same quantity. This quantity is determined by $\varepsilon_{1}$ to $\varepsilon_{2}$ ratio on operating waves. Therefore, half-sum of the direct values of two-colour temperatures is displaced relatively the $T$ on some quantity, which determines the error of method. This quantity depends on the $T$ and $\varepsilon_{1} / \varepsilon_{2}$. That is why for the error of method to be completely excluded it is necessary to use half-sum of reciprocal values of two-colour temperatures

$$
\frac{1}{T}=\frac{\frac{1}{S_{2 c}}+\frac{1}{S_{2 c}^{\prime}}}{2} \Rightarrow \mathrm{T}=\frac{2}{\frac{1}{S_{2 c}}+\frac{1}{S_{2 c}^{\prime}}}
$$


Equation (7) is the common pyrometric equation of the TCCT.

\section{Comparative investigations of the instrumental errors of the SWT, TCCT and also determined polychromatic (DPT) and spectral ratio (SR) thermometry}

As distinct from classical and known spectral thermometry, the SWT and the TCCT completely exclude the main components of method from the errors of optical temperature measurements. However, increase of the number of operating waves, i.e. transition to spectral thermometry results in instrumental errors increase. Such increase of instrumental errors excludes or restricts practical application of known multicolour thermometry solutions. Each method of the spectral thermometry has its own relationship between its metrological characteristics, number of operating waves and errors of measurements of one-colour temperatures on these waves. There is the following rule for spectral thermometry - the weaker this relationship, the more perfect the method. For example, for the DPT increase of operating waves number from 2 to 3 and 4 results in instrumental error increase in 3,4 and 8,8 times as well as in 3,5 and 14,0 times in spectral ranges $0,5-0,8 \mu \mathrm{m}$ and $0,5-1,4 \mu \mathrm{m}$ respectively ${ }^{[10]}$. These data were obtained under conditions of equal modules and signs of measurement errors of one-colour radiation temperatures. Therefore, investigations of the instrumental errors of spectral thermometry technologies are especially important for their compare and practical use.

The complex of comparative investigations of the instrumental metrological characteristics of the TCCT, 3-waves SWT and the $\mathrm{DPT}^{[10]}$ and also the classical spectral ratio (SR) thermometry is performed. Investigations were carried out for linearized in visible and near infrared ranges of spectrum (on $\lambda_{1}=0,7 \mu \mathrm{m}$ and $\lambda_{3}=0,9 \mu \mathrm{m}$ ) spectral distribution of emissivity of tungsten. Modules and signs of measurement errors of initial one-colour radiation temperatures were different for these investigations. Linearization provides exclusion of the component of method from measurement errors for the SWT and the TCCT. One-colour radiation temperatures $S_{1}, S_{2}$ and $S_{3}$ are the primary pyrometric information for spectral thermometry. Measurement error $\delta_{\text {meas. }}$ of one-colour radiation temperature is determined according to the formula

$\delta_{\text {meas. }}=\delta_{m}+\delta_{i}$,

where $\delta_{i}$ - instrumental component of the $\delta_{\text {meas. }}$;

$\delta_{m}-$ component of method of the $\delta_{\text {meas. }}$.

In our case $\delta_{m}=0$ and $\delta_{\text {meas. }}=\delta_{i}$. Therefore, here it is necessary to say only about instrumental errors of these initial one-colour temperatures. They determine instrumental errors of the spectral thermometry. As it was mentioned, instrumental metrological characteristics of modern energy one-colour thermometry are comparable or even exceed characteristics of thermoelectrical thermometry. That is why for investigations relative values of measurement errors of one-colour radiation temperatures equal 0,$2 ; 0,5$ and $1,0 \%$. These values significantly overlap real measurement errors of $S_{1}, S_{2}$ and $S_{3}$.

At the Table 3 the results of investigations of instrumental errors of the SWT and the DPT under conditions of equal signs and modules of measurement errors of one-colour radiation temperatures on $\lambda_{1}=0,7 \mu \mathrm{m}, \lambda_{2}=0,8 \mu \mathrm{m}$ and $\lambda_{3}=0,9 \mu \mathrm{m}$ are presented.

\begin{tabular}{|c|c|c|c|c|c|}
\hline \multirow{2}{*}{$\begin{array}{l}\text { Modules of } \\
\text { measurement errors } \\
\text { of one-colour } \\
\text { radiation } \\
\text { temperatures } \\
\left|\delta_{S 1}\right|=\left|\delta_{S 2}\right|=\left|\delta_{S 3}\right|, \% \\
\end{array}$} & \multirow{2}{*}{$\begin{array}{c}\text { Signs } \\
\text { distributions of } \\
\text { measurement } \\
\text { errors of } \\
\delta_{S 1}, \delta_{S 2}, \delta_{S 3}\end{array}$} & \multicolumn{2}{|c|}{ Result of measurement of $T$ : } & \multicolumn{2}{|c|}{ Instrumental errors: } \\
\hline & & $\begin{array}{l}\text { SWT } \\
\qquad T_{S W T}, \mathrm{~K}\end{array}$ & $\begin{array}{l}\text { DPT } \\
\qquad T_{D P T}, \mathrm{~K}\end{array}$ & $\begin{array}{l}\text { SWT } \\
\qquad \delta_{i . S W T}, \%\end{array}$ & $\begin{array}{l}\mathrm{DPT} \\
\quad \delta_{i . D P T}, \%\end{array}$ \\
\hline 0,2 & +++ & 1603,2 & 1603,2 & 0,20 & 0,20 \\
\hline 0,2 & - - - & 1596,8 & 1596,8 & $-0,20$ & $-0,20$ \\
\hline 0,5 & +++ & 1608,0 & 1608,0 & 0,50 & 0,50 \\
\hline 0,5 & $\ldots$ & 1592,0 & 1592,0 & $-0,50$ & $-0,50$ \\
\hline
\end{tabular}




\begin{tabular}{|l|l|l|l|l|l|}
\hline 1,0 & +++ & 1615,9 & 1615,9 & 0,99 & 0,99 \\
\hline 1,0 & --- & 1584,1 & 1584,1 & $-0,99$ & $-0,99$ \\
\hline
\end{tabular}

Table 3. Instrumental errors of the SWT and the DPT at $T=1600 \mathrm{~K}$

As we can see from the Table 3, instrumental errors of the SWT and the DPT are the same and do not exceed $\delta_{S 1}, \delta_{S 2}, \delta_{S 3}$. Under the most favourable conditions for DPT (when operating waves are equidistantly placed on the operating spectral range) and different signs of $\delta_{S 1}, \delta_{S 2}, \delta_{S 3}, \delta_{i . S W T}$ are more than $\delta_{i . S W T}$ in 1,1-28,2 times. The most exceeding (in 2,8-28,2 times) takes place for signs distribution $\left(_{+}++_{+}\right.$) and the least (in 1,1-2,0 times) - for distribution (- + -). These signs distributions are equivalent to maximal possible values of Kn. The SWT and the DPT are most sensitive for this parameter. If operating waves are placed non-equidistantly, instrumental errors of the DPT even greater exceed instrumental errors of the SWT.

Figure 7 illustrates dependences of instrumental errors of the TCCT and the SR thermometry for different modules and signs distributions of $\delta_{s 1}$ and $\delta_{s 3}$ on $\lambda_{1}$ and $\lambda_{3}$.

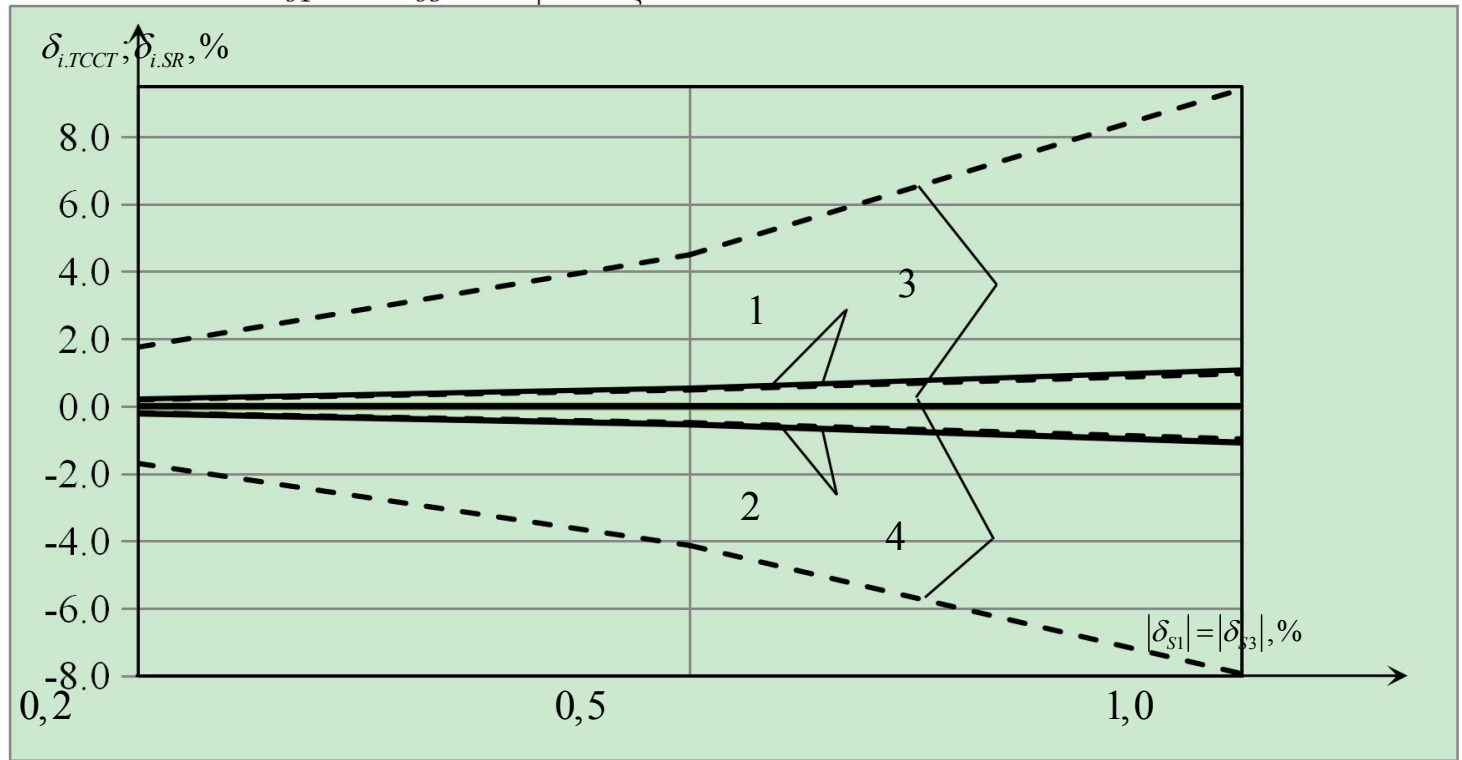

Figure 7; Dependences of instrumental errors of the TCCT and the SR thermometry for different modules and signs distributions of $\delta_{s 1}$ and $\delta_{s 3}$ :

$$
\begin{aligned}
& \text { - }- \text { - sCCT } ; \text { - - - }-\mathrm{SR} ; \\
& 2 \text { - signs distribution }(++) ; \\
& 3 \text { - signs distribution }(--) ; \\
& 4 \text { - signs distribution }(+-) ;
\end{aligned}
$$

For the same signs and modules of $\delta_{s 1}$ and $\delta_{s 3}, \delta_{i . S R}$ are lower, than $\delta_{i . T C C T}$ by $0,03-0,11 \%$. With increase of modules of $\delta_{s 1}$ and $\delta_{s 3}$ from 0,2 to $1,0 \% \delta_{i . S R}$ and $\delta_{i . T C C T}$ increase in 5,1 and 4,1 times, respectively.

For the same signs and modules of $\delta_{s 1}$ and $\delta_{s 3}, \delta_{i . T C C T}$ are higher than measurement errors of one-colour radiation temperatures by $0,02-0,08 \%$. When the signs are different $S_{2 c}$ and $S_{2 c}^{\prime}$ shift symmetrically relatively to the object temperature and $\delta_{i . T C C T}$ become negligible small (do not exceed $0,02 \%$ for $\left|\delta_{s 1}\right|=\left|\delta_{s 3}\right|=1,0 \%$ ). At the same time $\delta_{i . S R}$ reach $1,7 \%$ (for $\left|\delta_{s 1}\right|=\left|\delta_{s 3}\right|=0,2 \%$ ) and $9,4 \%$ (for $\left|\delta_{s 1}\right|=\left|\delta_{s 3}\right|=1,0 \%$ ).

In accordance with the Table 3 and the Figure 7 the SWT and the TCCT show the best results for instrumental errors. For the same modules and signs of $\delta_{s 1}, \delta_{s 2}, \delta_{s 3}$, the instrumental errors of the SWT are lower than the TCCT ones by $0,02-0,09 \%$. When signs of the $\delta_{s 1}, \delta_{s 2}, \delta_{s 3}$ are different, instrumental errors of the TCCT are close to zero and instrumental errors of the SWT exceed 2,9\%. These different signs can take place due to different spectral transmissivity of intermediate medium. Mentioned quantitative estimations demonstrate significant metrological instrumental advantages of the TCCT comparing to other considered spectral thermometry technologies. 


\section{Conclusions}

Thus, as a result of comparative investigations, it was established, that symmetric-wave and two-colour compensating pyrometry have higher, in comparison with known solutions, metrological characteristics. It was proved that errors of method of spectral linear symmetric-wave thermometry under conditions of nonlinear spectral distribution of emissivity are in 2,3; 4,9-13,2 and 3,1-3,6 times smaller, than errors of method of known spectral and also classical energy and spectral ratio thermometry respectively. The errors of method of linear symmetric-wave thermometry equal zero for $\Delta \lambda \leq 0,05 \mu \mathrm{m}$. Universal symmetric-wave and two-colour compensating thermometry allow to exclude completely the errors of method from the errors of optical temperature measurements.

Under the most probable conditions of industrial measurements with different signs of one-colour radiation temperatures measurements errors instrumental errors of spectral linear symmetric-wave thermometry are in 1,1-28,2 times smaller, than instrumental errors of determined polychromatic thermometry. Instrumental errors of two-colour compensating thermometry are negligible small in comparing of these ones for the symmetric-wave and moreover for the classical spectral ratio thermometry.

This instrumental advantage of the two-colour compensating thermometry in comparison with the symmetric-wave thermometry takes place under conditions of different signs of measurements errors of one-colour radiation temperatures. Instrumental errors of the symmetric-wave and two-colour compensating thermometry are almost equal in the case of the same signs.

Spectral thermometry technologies are based on remote quantitative determination of the spectral emissivity distributions of controlled objects. Using these distributions and well-known relationships between the spectral coefficients of emission, absorption, reflection and transmission macro-optical characteristics of these objects can be determined and remote identification of them can be performed. Therefore, new thermometry technologies can also be used for special and aerospace researches and control.

\section{References}

1. Zhukov LF, Petrenko DA, Kornienko AL. History, state and development perspectives of temperature measurements in metallurgy. Metal and foundry of Ukraine 2012; (7): 27-34.

2. Zhukov LF, Kornienko AL. New technologies of symmetric-wave multicolour thermometry and their metrological characteristics. Journal of Engineering Physics and Thermophysics 2014; 87 (2): 489-497.

3. Sokolov AV. Optical properties of metals. Moscow: Fizmatgiz, 1961.

4. Zhukov LF. Investigation of spectral distribution of heat radiation of iron-carbon alloys. Casting processes 1998; (3-4): 154-157.

5. Zhukov LF. Investigation of temperature regularities of heat radiation of iron-carbon melts. Casting processes 1996 (4): 9-19.

6. Zhukov LF, Kornienko AL. New technologies of multicolour symmetric-wave thermometry. Saarbrucken: Lambert Academic Publishing, 2014.

7. Zhukov LF, Kornienko AL. The way of temperature measurement. Patent of Ukraine No 76096, IPC G01J 5/00. Published 25.12.2012, bulletin №24.

8. Thomas LK. Journal of Scientific Instruments 1968; Ser. 2, 1: 311.

9. Zhukov LF, Petrenko DA, Kornienko AL. The way of temperature measurement. Patent of Ukraine No 116015, IPC G01J 5/00. Published 10.05.2017, bulletin №9.

10. Snopko VN. The bases of pyrometry methods using the spectrum of heat radiation. Minsk: Institute of physics named after B. I. Stepanov NASB, 1999. 